associations between social isolation, loneliness, socioeconomic position, health behaviours and symptoms of poor mental health further obscure our understanding of potential pathways. This study therefore aimed to explore time-varying associations between social isolation, living alone and loneliness and neuro-immune markers in older adults, whilst accounting for a comprehensive range of confounders.

Methods We analysed blood samples from 8780 adults aged 50 and above from the English Longitudinal Study of Ageing (ELSA), a nationally-representative longitudinal cohort study, across three waves of data collection: 2004/5, 2008/9 and $2012 / 2013$. At baseline, the sample was 55\% female, $66.4 \%$ married or in a partnership and $44.2 \%$ had no or basic qualifications. Multiple imputation was used to account for missing data. Fixed effects modelling was used to estimate independent relationships between loneliness, social isolation, living alone and levels of three inflammatory markers; fibrinogen, white blood cell (WBC) count and C-reactive protein (CRP), and the neuro-inflammatory regulator insulin like growth factor-1 (IGF-1).

Results ELSA participants who experienced an increase in social engagement were found to have decreased levels of the inflammatory markers fibrinogen (fixed effects coefficient: -0.007 [confidence interval: $-0.015-0.001])$, WBC $(-0.012[-0.021--0.003])$ and CRP $(-0.040[-0.078-$ $-0.002])$. Similarly, living status was inversely associated with fibrinogen $(-0.057[-0.097--0.018])$, WBC $(-0.098$ $[-0.147--0.048])$ and CRP $(-0.238[-0.416--0.060])$. By contrast, decreased loneliness was associated with increased IGF-1 $(0.133[0.026-0.240])$. The findings were independent of time-invariant factors such as gender, medical history, unobserved aspects of social position, and genetics, and time-varying factors such as income, physical health, health behaviours, and depressive symptoms. The results were maintained in sensitivity analyses that accounted for BMI, gender interaction, survey weighting and exclusion of imputed values.

Conclusion Whilst causality cannot be assumed in observational studies, the results suggest that being alone and feeling alone are distinct biosocial stimuli. Assuming the methodology sufficiently accounted for confounding factors, this interpretation is especially relevant to the current social prescribing and healthy ageing movements.

\section{OP38 CO-MORBIDITY AND CO-DEVELOPMENT OF BMI AND MENTAL HEALTH FROM CHILDHOOD TO MID- ADULTHOOD IN TWO NATIONAL BIRTH COHORT STUDIES}

\begin{abstract}
${ }^{1,2} \mathrm{AR}$ Khanolkar, ${ }^{3} \mathrm{CA}$ Hardman, ${ }^{4} \mathrm{D}$ Bann, ${ }^{5} \mathrm{~W}$ Johnson, ${ }^{1,4} \mathrm{P}$ Patalay*. ${ }^{1} \mathrm{MRC}$ Unit for Lifelong Health and Ageing, University College London, London, UK; ${ }^{2}$ Institute for Environmental Medicine, Karolinska Institutet, Stockholm, Sweden; ${ }^{3}$ Department of Psychological Sciences, University of Liverpool, Liverpool, UK; ${ }^{4}$ Centre for Longitudinal Studies, University College London, London, UK; ${ }^{5}$ School of Sport, Exercise and Health Sciences, Loughborough University, Loughborough, UK
\end{abstract}

\subsection{6/jech-2020-SSMabstracts.38}

Background Obesity and poor mental health are increasingly common global chronic conditions likely to originate in childhood and have a propensity to track across the lifecourse. Their co-morbidity, co-development and longitudinal associations across the lifecourse are not well known, including whether these might be changing across cohorts.
The main aim was to study co-morbidity and co-development of obesity and poor mental health across childhood and into mid-adulthood using data from two national birth cohorts.

Methods This study analysed BMI and mental health data from participants that attended any one of the ages $11 / 10$, 16, 23/26, 33/34 and 42 assessments from the 1958 National Child Development Study (NCDS58) and the 1970 British Cohort Study (BCS70) [total $\mathrm{N}=30,868,51 \%$ males]. Mental health was based on symptoms of anxiety and depression assessed by questionnaires answered by participants (or their parents during childhood).

Odds of co-occurrence of obesity and poor mental health were analysed using multivariable logistic regression at each age for the entire sample and separately for each cohort, adjusting for sex, and childhood and adulthood socioeconomic position.

Latent spline growth models were used to assess the codevelopment of BMI and mental health (associations between intercepts and slopes) across all 5 ages adjusting for sex and socioeconomic indicators. Growth models were also run restricting to adulthood sweeps only. Missing data was addressed using multiple imputation.

Results Obesity and poor mental health were found to be comorbid only in adulthood (adjusted odds ratio (OR) 1.31 [95\% CI 1.19-1.43] at age 23/26, 1.22 [1.13-1.32] at age 34/ $33,1.2[1.11-1.30]$ at age 42$)$. Co-morbidity was more likely in the BCS70 compared to NCDS58 cohort (for example, OR 1.22 [1.09-1.38] vs. 1.08 [0.97-1.2] at age 42 respectively).

Growth modelling revealed that BMI development across all 5 ages was predicted by mental health development (for example, a unit change in mental health slope was associated with 0.15 unit change in the BMI slope, [95\% CI $0.13-$ $0.18])$. This was observed to be even stronger in adulthoodonly models (adjusted standardised coefficient 0.56, 0.450.66). Estimates were similar in strength in both cohorts (0.52, [0.38-0.66] in NCDS58 and 0.54, [0.39-0.68] in BCS70) and sexes (0.5 [0.36-0.64], males and 0.61 [0.460.76], females). BMI was not found to predict mental health development in any model.

Conclusion Findings indicate that obesity and poor mental health are more likely to co-occur in adulthood compared to childhood and the co-occurrence is more likely in the more recently born BCS70 cohort.

\section{Thursday 10 September Pregnancy I}

\section{OP39 ANALYSIS OF SPONTANEOUS, USER-GENERATED DATA ABOUT GESTATIONAL DIABETES ON ONLINE FORUMS: IMPLICATIONS FOR DIABETES PREVENTION}

${ }^{1} \mathrm{CE}$ Eades, ${ }^{1} \mathrm{~K}$ Clarke, ${ }^{1} \mathrm{DM}$ Cameron, ${ }^{2} \mathrm{~N}$ Coulson, ${ }^{1} \mathrm{JMM}$ Evans*. ${ }^{1}$ Faculty of Health Sciences and Sport, University of Stirling, Stirling, UK; ${ }^{2}$ Faculty of Medicine and Health Sciences, University of Nottingham, Nottingham, UK

\subsection{6/jech-2020-SSMabstracts.39}

Background Perceptions of gestational diabetes mellitus (GDM) and future risk of Type 2 diabetes (T2D) have previously been explored in traditional qualitative research interviews among women who have had GDM. These findings 